\title{
Comments on the ESC 2015 Guidelines for the Management of Infective Endocarditis
}

\author{
Comentarios a la guía ESC 2015 sobre el tratamiento de la endocarditis infecciosa
}

SEC Working Group for the ESC 2015 Guidelines on the Management of Infective Endocarditis, Expert Reviewers for the ESC 2015 Guidelines on the Management of Infective Endocarditis, and the SEC Guidelines Committee ${ }^{\triangleleft}$

\section{INTRODUCTION}

The guidelines of the European Society of Cardiology (ESC) are endorsed by the Spanish Society of Cardiology (Sociedad Española de Cardiología, [SEC]) and are translated into Spanish for publication in Revista Española de Cardiologia. Every updated version of the guidelines is accompanied by a commentary article written in accordance with the aims and methods recommended by the Clinical Practice Guidelines Committee of the SEC. ${ }^{1}$ The present article, drafted by a team of experts appointed by the SEC Committee, discusses the new ESC guidelines for the management of infective endocarditis. ${ }^{2}$ Another group of experts, recruited by the Clinical Cardiology Section of the SEC and including specialists in cardiac imaging, have made important contributions to the document presented here.

The 2015 Guidelines are an update of those published in 2009. Over the intervening 6 -year period, there have been important advances in the field that justify publication of an updated version: a) since the recommendation to restrict prophylaxis to high-risk patients in high-risk situations, several large registries have appeared, with different conclusions, and it seems appropriate that the ESC renew its position in this regard; $b$ ) a new approach is described, involving specialized units in which experts in different disciplines collaborate in the care of endocarditis patients, and the criteria for referral to these units is specified; $c$ ) a randomized study ${ }^{3}$ comparing medical treatment with surgical treatment has emerged, although the related evidence has not changed substantially because of the limitations described; $d$ ) there has been considerable progress in the application of imaging techniques to the diagnosis of endocarditis and these important changes have led to new criteria for diagnosing the disease; and e) substantial modifications have been made in the proposed antibiotic therapy, mainly focused on avoiding toxicity.

As was the case of the 2009 guidelines, there is little high-level evidence to uphold the recommendations in the current update, which contains 1 Level A recommendation, 48 Level B (half referring to antibiotic therapy), and 50 Level C (13 referring to antibiotic

\section{SEE RELATED ARTICLE:}

http://dx.doi.org/10.1016/j.rec.2015.12.002, Rev Esp Cardiol. 2016;69:69.e1-e49.

${ }^{\ominus} \mathrm{A}$ complete list of the authors who contributed to the article is provided in the Appendix.

Corresponding author: Departamento de Cardiología, Hospital Clínico Universitario, Avda. Ramón y Cajal 3, 47005 Valladolid, Spain

E-mail address: asanroman@secardiologia.es (A. San Román) therapy). Nonetheless, the guidelines are an indispensable tool for specialists interested in infective endocarditis. The topics are presented in an instructive manner and include all relevant information on this disease.

As an additional strength, the guidelines are endorsed by the European Association for Cardio-Thoracic Surgery and the European Association of Nuclear Medicine, and the contributing experts include a representative from the European Society of Clinical Microbiology and Infectious Diseases. In addition, various associations belonging to the ECS have participated.

The following sections discuss each of the areas covered by the updated guidelines, with an emphasis on the new information provided, its positive and controversial aspects, and the implications for our daily clinical practice.

\section{PREVENTION}

The section on prevention proposes general measures for all patients (not only those at high risk) to decrease the incidence of healthcare-related infective endocarditis, and particularly targets hospitalized patients undergoing various procedures. In this line, it includes a new recommendation that has a considerable impact on clinical practice: preoperative screening for nasal carriage of Staphylococcus aureus should be carried out in all patients and carriers should receive preoperative treatment. This is the only recommendation with Level A evidence. Another interesting innovation is the recommendation to eliminate potential septic foci, mainly dental sources, before scheduled surgical procedures. Lastly, patients with transcatheter prostheses are included in the groups requiring prophylaxis, as the associated incidence of infective endocarditis is similar to that related to prosthetic valves, and mortality is high.

The most important part of this section discusses the reasons why the prophylaxis recommendations set out in the 2009 guidelines have been maintained in the updated document, despite publication of important articles that might point to the need for changes. For example, a recent study in the United Kingdom reported an increase in the incidence of infective endocarditis since 2008 when more restrictive criteria than those used in the remaining European countries were applied and prophylaxis was reduced; nonetheless, a study carried out in France (where restrictions were also applied) reported no such increase. Moreover, microbiological data were not included in the British study; hence, it is impossible to know whether prophylaxis would have avoided the cases reported. Lastly, the statistical analysis in the study has been criticized because a cutoff at 
any date between 2003 and 2010 would have yielded a similar significant increase in endocarditis incidence when using the authors' statistical approach. ${ }^{4}$ The guidelines mention several studies from the United States that have also reported contradictory findings. In a recent article from that country (not mentioned), there was no increase in infective endocarditis due to viridans group streptococci following reductions in prophylaxis according to the guidelines, even though prophylactic measures are effective against these microorganisms. ${ }^{5}$ In short, it seems a wise choice to maintain the restrictive approach to antibiotic prophylaxis, with no changes in the previous recommendations.

\section{SPECIALIZED ENDOCARDITIS UNITS}

This is one of the most innovative aspects of the new guidelines. This section contains an explanation of why endocarditis requires multidisciplinary assessment, when to refer a patient with endocarditis to a specialized unit in a reference center, what characteristics the reference center should have, and what role the specialized unit has in this disease. Several studies, some performed in Spain, ${ }^{6}$ have reported reductions in mortality that lend support to these recommendations. The multidisciplinary team should choose the type, duration, and follow-up of antibiotic therapy, decide on the need for surgery, and establish a follow-up schedule, including the timing of imaging assessments.

Although hospital cardiology departments are always involved in echocardiographic diagnosis of these patients, they may not be directly responsible for the patients' care or for establishing the treatment. The new recommendations should serve as a stimulus to implement specialized units in reference hospitals where cardiologists will have a key role, and not only as imaging experts. In Spain there is considerable experience with this type of health care design. ${ }^{7}$

\section{DIAGNOSIS}

This section discusses the clinical and analytic findings in infective endocarditis, the importance of the 2 diagnostic pillars, imaging and microbiology, and the diagnostic criteria.

The guidelines continue to cite echocardiography as the technique of choice for the diagnosis of endocarditis. As explained in detail, transthoracic echocardiography is the first-line diagnostic technique, but its sensitivity is limited in the assessment of prosthetic valves and detection of periannular complications. For this reason, transesophageal echocardiography is indicated to evaluate the presence of complications in patients with prosthetic valves and positive or highly suspicious findings on transthoracic echocardiography. Furthermore, as in the previous guidelines, a transesophageal study is recommended when there is a high index of suspicion for the disease on clinical grounds, but (as in previous guidelines) "high clinical suspicion of endocarditis" is not defined (nor is "suspicion" defined for the indication of transthoracic echocardiography). As there are no clear criteria in this respect, some echocardiography laboratories may be too tolerant and have to bear an extra workload, whereas others may be too restrictive and miss some cases of the disease. One innovation is that prosthetic valve dehiscence and aneurysm detected on echocardiography are included as major diagnostic criteria; these are uncommon but not exceptional findings. Another aspect discussed is the timing for a new transesophageal echocardiogram when a high index of suspicion persists: the new recommendation for this assessment is at 5 to 7 days, instead of the 7 to 10 days recommended in the previous guidelines. Finally, 3D transesophageal echocardiography is introduced in the update as a supplement to the conventional technique.

New imaging techniques (magnetic resonance, computed tomography, nuclear techniques) have been included in the diagnostic process with 3 possible objectives: a) detection of abscesses; b) detection of inflammatory activity around prosthetic valves
3 months after surgery, and c) detection of cerebral and peripheral embolisms. The first 2 are considered major diagnostic criteria and the third a minor criterion. Undoubtedly, these techniques decrease the number of endocarditis cases classified as possible and they improve the diagnostic process, mainly in endocarditis associated with prosthetic valves and implantable cardiac devices. ${ }^{8}$ In the latter case, however, the authors did not include the results of the new imaging techniques as diagnostic criteria because of the lack of related evidence.

\section{PROGNOSTIC ASSESSMENT AT HOSPITAL ADMISSION}

This short section is very important from the clinical standpoint. It is conscientiously positioned between the diagnosis and treatment sections (not at the end, where prognosis is usually placed) to emphasize that the initial prognostic evaluation should be made with the data available in the first few days following admission, all of which have been covered in the section on the diagnosis. The message that an initial prognostic assessment based on clinical, echocardiographic, and microbiologic data is essential to determine the best therapeutic strategy was already included in the previous guidelines. This update additionally mentions the prognostic importance of persistently positive blood cultures at 48 to 72 hours after initiation of appropriate antibiotic therapy.

\section{ANTIBIOTIC THERAPY}

The authors recognize that the recommendations for the majority of antibiotic regimens have been based on consensus, and that the optimal treatment for staphylococcal endocarditis and the empirical treatment are still under debate.

Table

Changes Established in Antibiotic Therapy

Restrictions in aminoglycoside therapy:

- Not recommended for native valve Staphylococcus aureus endocarditis

- Administration in a single daily dose

- Two weeks for enterococci and penicillin-resistant streptococci

- Alternative regimen for Enterococcus faecalis endocarditis: ampicillin and ceftriaxone ${ }^{\mathrm{a}}$

New cutoffs to define penicillin-susceptible (MIC $\leq 0.125 \mathrm{mg} / \mathrm{L}$ ), intermediate resistant (MIC $0.25-2 \mathrm{mg} / \mathrm{L}$ ), and resistant (MIC > $4 \mathrm{mg} / \mathrm{L}$ ) streptococci

New cutoffs to define penicillin-susceptible (MIC $\leq 0.06 \mathrm{mg} / \mathrm{L}$ ), intermediate resistant (MIC 0.125-2 mg/L), and resistant (MIC $\geq 4 \mathrm{mg} / \mathrm{L}$ ) pneumococcal strains

Ceftriaxone is an option in endocarditis caused by relatively penicillin resistant (MIC 0.125-2 mg/L) oral streptococci and the Streptococcus bovis group, preferably by outpatient administration

Desensitization in patients with methicillin-susceptible $S$. aureus endocarditis and allergy to beta lactams

High-dose vancomycin for staphylococcal endocarditis: $30-60 \mathrm{mg} / \mathrm{kg} / \mathrm{d}$, divided into 2-3 doses (trough level $\geq 20 \mathrm{mg} / \mathrm{L}$ )

High-dose daptomycin to treat $S$. aureus endocarditis in patients allergic to penicillin and infections caused by methicillin-resistant strains ${ }^{\mathrm{b}}$

Rifampicin for prosthetic valve endocarditis following 3-5 d of effective treatment at a lower dose (900-1200 mg/d, divided into 2-3 doses)

Six weeks' duration of endocarditis therapy for prosthetic valve infection due to HACEK group microorganisms

Doxycycline and hydroxychloroquine for Coxiella burnetii ( $Q$ fever) endocarditis

High-dose echinocandins to treat endocarditis due to Candida spp.

Voriconazole (combined or not with echinocandins or amphotericin B) to treat endocarditis caused by Aspergillus

New initial empirical therapy regimen for native valve infection: ampicillin, cloxacillin, and gentamicin

${ }^{a}$ This combination enables treatment of all cases of $E$. faecalis endocarditis with no risk of toxicity and regardless of the degree of aminoglycoside resistance.

${ }^{\mathrm{b}}$ Administration with a second antibiotic is proposed to increase the activity and avoid the emergence of resistance. 
The new guidelines establish numerous changes in antibiotic therapy with respect to the previous recommendations (Table). We highlight those that have clinical implications. First, aminoglycoside administration is restricted to avoid associated toxicity, as there is little evidence to sustain its use. These agents are not recommended for native valve staphylococcal endocarditis and, if needed, they should be administered in a single daily dose. In addition, the guidelines accept shortening the duration of therapy to 2 weeks in enterococcal endocarditis (Class IIa recommendation). The combination of ampicillin and ceftriaxone is proposed instead of amoxicillin and gentamicin to treat endocarditis caused by Enterococcus faecalis. This new combination is the treatment of choice for infections caused by strains with high-level aminoglycoside resistance.

Rifampicin is recommended only for foreign body infections, after 3 to 5 days of effective antibiotic therapy and after resolution of bacteremia. This is a troublesome point because there is very little evidence regarding what drugs should be given and whether therapy should be delayed for several days (the previous guidelines did not establish this delay).

Daptomycin, fosfomycin, and netilmicin are considered alternative therapies to the indicated treatment because they are not available in all European countries. Daptomycin is indicated for treating Staphylococcus aureus endocarditis in patients allergic to penicillin and for methicillinresistant staphylococci. The drug should be given at high doses and combined with a second antibiotic to increase its activity and avoid the emergence of resistance. If daptomycin is used, creatine kinase should be monitored on a weekly basis. Irrespective of these considerations, the evidence sustaining daptomycin use is still weak. Most cohort studies on daptomycin for the treatment of staphylococcal endocarditis have included relatively small, heterogeneous groups of patients previously treated with vancomycin. At present it is difficult to establish the optimum regimen. Of note, the antibiotic recommendations for staphylococcal endocarditis are based on studies investigating infections caused by $S$. aureus. Because there are very few studies on plasma coagulase-negative staphylococci, it is often assumed that these microorganisms will have the same microbiological patterns as S. aureus, an assumption that may not be correct.

Desensitization should be attempted in stable, beta lactam-allergic patients with endocarditis caused by methicillin-susceptible $S$. aureus, as vancomycin is less effective than beta lactams for this infection.

An important point related to our geographical area that may not have been fully taken into account in the new guidelines is that a combination of hydroxychloroquine plus doxycycline is proposed for the treatment of endocarditis caused by Coxiella burnetii (agent of $\mathrm{Q}$ fever), but the combination of quinolones plus doxycycline for this purpose has disappeared. In southern European countries, hydroxychloroquine administration may be a problem because of potential phototoxicity. There is considerable experience with quinolones plus doxycycline for treating $\mathrm{Q}$ fever endocarditis in Spain, and the results have been favorable. ${ }^{9}$

Finally, a new regimen has been proposed for initial empirical treatment of native valve endocarditis: ampicillin, cloxacillin, and gentamicin. The addition of cloxacillin seems particularly appropriate as it has greater activity against staphylococci than vancomycin with little associated toxicity.

\section{COMPLICATIONS AND SURGICAL TREATMENT}

The guidelines first discuss the main complications-heart failure, uncontrolled infection, and embolism-which are indications for surgery in many cases. The others covered include neurological complications, infectious aneurysms, splenic complications, myocarditis, pericarditis, conduction disorders, rheumatic complications, and renal complications.

In the new guidelines, the indications for surgery have been simplified. Instead of having 2 sections, 1 for patients with native valve infections and 1 for those with prosthetic valves, as in the former guidelines, the indications are summarized in a single table divided into 3 sections: $a$ ) patients with heart failure; $b$ ) patients whose antibiotic treatment does not achieve control of the infection, and $c$ ) patients referred for surgery with the main objective of preventing embolic events.

As in the previous guidelines, the authors recognize that most patients with heart failure will require surgery. There is a new proposal in the section on uncontrolled infection: Surgery is recommended (Class IIa) in patients with persistent bacteremia (positive blood cultures) despite appropriate antibiotic therapy and after exclusion of other septic foci. The guidelines mention that it is not necessary to wait 7 to 10 days, as recommended in the previous version, and that positive blood culture 2 to 3 days following initiation of adequate antibiotics is sufficient.

There are 2 new indications for surgery to prevent embolic events. In the first, patients with left-sided native valve endocarditis, severe valvular regurgitation, and vegetations $>10 \mathrm{~mm}$ are considered candidates for surgery, particularly if they are at low surgical risk and there is a good possibility that the valve can be repaired. This recommendation is the result of the only randomized study performed in patients with endocarditis. ${ }^{3}$ Although it has many limitations (practically a single-center design, small number of patients at low risk, and microorganisms whose microbiological profile differs from that seen in most recent series), it is an important article showing that randomized studies can be carried out in diseases as complex as endocarditis.

Surgery can also be considered in patients with exceptionally large vegetations $(>3 \mathrm{~cm})$, as a high incidence of neurological complications has been documented in a Spanish multicenter study including these patients. ${ }^{10}$ The guidelines mention other risk factors to take into consideration when determining the risk of embolism, and attempts have been made to include some of them in risk prediction scores. Understandably, when surgery is indicated to prevent embolisms, it should be carried out urgently, 2 to 3 days after the start of antibiotic therapy. This point should be stressed because embolic events occur most often in the first week after initiating antibiotics.

With regard to neurologic complications, which are important because of their associated morbidity and mortality, there are no large changes relative to the previous guidelines. Many patients with these complications will continue to be candidates for valve surgery. It is difficult to decide on the timing of the procedure in this situation, as the optimal time interval between the neurologic event and surgery has not been established. For this reason we believe that the change from a Class I to a Class Ila recommendation in patients with intracranial bleeding is appropriate, as a recent study reported that these patients can be referred for surgery within 2 weeks (instead of 1 month as the guidelines recommend) with a low risk of neurological deterioration. ${ }^{11}$ Neurologists and neurosurgeons should be involved in the decision for surgery and other treatments in patients with neurological complications.

Among the complications covered, the present guidelines specifically discuss heart rhythm disturbances (atrial fibrillation) and conduction disturbances (atrioventricular block). These abnormalities are common in endocarditis patients and were not included in the previous guidelines. With regard to rheumatic complications, the utility of positron emission tomography with ${ }^{18} \mathrm{~F}$ fluorodeoxyglucose combined with computed tomography $\left({ }^{18} \mathrm{~F}\right.$ FDG PET/CT) is highlighted in the diagnosis and monitoring of spondylodiscitis, a common complication in this disease. Nonetheless, recurrence is rare and there is no evidence supporting monitoring with these techniques, which involve considerable radiation exposure.

Another new section, absent in the previous guidelines, deals with determination of operative risk. The guidelines state the importance of risk estimation and cite 2 risk scores specific to patients with infectious endocarditis that are better predictors of mortality 
following surgery for active endocarditis than the EuroSCORE II, which was not designed for this scenario.

\section{PRONOSIS FOLLOWING DISCHARGE}

Two periods related to patient follow-up after hospital discharge are mentioned in the new guidelines: short-term follow-up and longterm prognosis. The most frequent complications after hospital discharge are heart failure secondary to a severe residual valvular lesion, and less commonly, recurrent infection. There are very few changes with respect to the previous guidelines. For example, chronic dialysis has been added to the list of factors associated with an increased risk of recurrence. Once antibiotic therapy has finished and the patient is discharged, the indications for valve surgery are the same as for any other patient with valvular heart disease.

\section{SPECIFIC SITUATIONS}

While there are clear reasons to deal separately with certain infections, such as intracardiac device-related infective endocarditis and right-sided endocarditis, we do not consider there is a need for a specific section on endocarditis in the intensive care unit. This is simply endocarditis in severely ill patients with septic, hemodynamic, neurologic, or other complications. It adds nothing to what is written in the other sections. The current guidelines do not include a section dedicated to endocarditis in the elderly population, present in previous versions, possibly because the approaches applied would not vary according to the age of the patient.

Emphasis is placed on the added value of nuclear medicine and other imaging techniques, especially ${ }^{18} \mathrm{~F}-\mathrm{FDG}$ PET/CT and cardiac CT, to attain a definitive diagnosis in patients with a prosthetic valve and normal echocardiography findings. Because of the high sensitivity and negative predictive value of ${ }^{18} \mathrm{~F}-\mathrm{FDG} \mathrm{PET} / \mathrm{CT}$, this technique complements echocardiography in patients with suspected prosthesis-related endocarditis and an inconclusive echocardiogram. Nonetheless, the technique has limitations: The physiologic uptake occurring in normal myocardium may make interpretation of the results difficult, the duration of antibiotic therapy prior to the test may affect the results, the normal postoperative inflammatory response may cause false-positive findings in recently operated patients, and other diseases in addition to endocarditis can lead to increased ${ }^{18} \mathrm{~F}$-FDG uptake. Therefore, further studies are needed, including studies in other patient populations (with a lower prevalence of endocarditis), to validate the accuracy of this technique and establish definite recommendations.

The section on endocarditis occurring in patients with cardiac implantable electronic devices mentions that it may still be difficult to determine whether the infection is local and limited to the generator pocket or is extended and considered device-related endocarditis. Although the recommendation is not as strong as for prosthetic valves (Class IIb), emphasis is placed on the use of ${ }^{18} \mathrm{~F}-\mathrm{FDG}$ $\mathrm{PET} / \mathrm{CT}$ or radiolabeled leukocyte scintigraphy to reach a definite diagnosis. Once again, the diagnostic accuracy of these techniques for detecting infection is not well established in this scenario. Prolonged antibiotic therapy and complete hardware removal are recommended for treating the infection. Percutaneous removal of the system is feasible in most patients, and this mode is recommended even in patients with large vegetations. Complete extraction of the device is also recommended in infections presumed to be limited to the generator pocket.

The sections on right-sided endocarditis and congenital heart disease show very few changes with respect to the previous guidelines, and there are no changes in the recommendations for surgery.

In the new guidelines, urgent surgery is recommended for pregnant women with valve regurgitation and heart failure, a rare situation. Surgery in these patients is associated with high mortality for the mother and fetus; thus, it is a difficult decision that should be individualized and reached by consensus with the patient and her gynecologist.

The sections on nonbacterial thrombotic endocarditis and endocarditis associated with cancer are new and, in our opinion, very appropriate. It is particularly important to differentiate between patients with nonbacterial thrombotic endocarditis and those with culture-negative infectious endocarditis. Nonbacterial thrombotic endocarditis should be suspected in patients with cancer or other diseases associated with this condition, negative blood cultures, and multiple systemic emboli. The vegetations are characteristically small, valvular regurgitation tends to be mild, and there is usually no periannular extension of the infection (abscesses, pseudoaneurysms, or fistulas). Therapy for the infection consists in treating the underlying condition.

\section{CONFLICTS OF INTEREST}

None declared.

\section{APPENDIX: AUTHORS}

SEC Working Group for the ESC 2015 Guidelines on the Management of Infective Endocarditis: J. Alberto San Román (coordinator), Isidre Vilacosta (coordinator), Juan Carlos Castillo Domínguez, Nuria Fernández Hidalgo, Carlos González Juanatey, Javier López, and Jacobo Silva.

Expert Reviewers for the ESC 2015 Guidelines on the Management of Infective Endocarditis: María Castiñeira Busto, José Alberto García Lledó, Juan José Gómez Doblas, José Julio Jiménez Nacher, Domingo Marzal Martín, José Luis Moya Mur, Joan Carles Paré Bardera, and Alejandra Vaello Paños.

SEC Guidelines Committee: Manuel Anguita (president), Ángel Cequier (secretary), Fernando Alfonso, Lina Badimón, José Antonio Barrabés, Ignacio Fernández Lozano, José Juan Gómez de Diego, Luis Rodríguez Padial, José Alberto San Román, Pedro Luis Sánchez Fernández, Juan Sanchis, and Alessandro Sionis.

\section{REFERENCES}

1. Anguita M, Fernández-Ortiz A, Worner F, Alonso A, Cequier A, Comín J, et al. La Sociedad Española de Cardiología y las guías de práctica clínica de la ESC: hacia una nueva orientación. Rev Esp Cardiol. 2011;64:795-6.

2. Habib A, Lancellotti P, Antunes MJ, Bongiorni MG, Casalta JP, Del Zotti F, et al. 2015 ESC Guidelines for the management of infective endocarditis: The Task Force for the management of Infective endocarditis of the European Society of Cardiology. Eur Heart J. 2015. doi:10.1093/eurheartj/ehv319

3. Kang DH, Kim YJ, Kim SH, Sun BJ, Kim DH, Yun SC, et al. Early surgery versus conventional treatment for infective endocarditis. N Engl J Med. 2012;366:2466-73.

4. Iung $B$, Tubiana $S$, Alla $F$, Lavielle $M$. Infective endocarditis and antibiotic prophylaxis. Lancet. 2015;386:529-30.

5. DeSimone DC, Tleyjeh IM, Correa de Sa DD, Anavekar NS, Lahr BD, Sohail MR, et al Incidence of infective endocarditis due to viridans group streptococci before and after the 2007 American Heart Association's Prevention Guidelines: an extended evaluation of the Olmsted County, Minnesota, population and nationwide inpatient sample. Mayo Clin Proc. 2015;90:874-81.

6. Carrasco-Chinchilla F, Sánchez-Espín G, Ruiz-Morales J, Rodríguez-Bailón I, Melero-Tejedor JM, Ivanova-Georgieva R, et al. Influencia de una estrategia de alerta multidisciplinaria en la mortalidad por endocarditis infecciosa izquierda. Rev Esp Cardiol. 2014;67:380-6.

7. Mestres CA, Paré JC, Miró JM. Organización y funcionamiento de un grupo multidisciplinario de diagnóstico y tratamiento de la endocarditis infecciosa: perspectiva de 30 años (1985-2014). Rev Esp Cardiol. 2015;68:363-8.

8. Moragas M, Sánchez R, Soler M, Cozar MP, Riera E, García JR. Detección de infección del electrodo del DAI mediante tomografía por emisión de positrones-tomografía computarizada con ${ }^{18} \mathrm{~F}$-fluorodesoxiglucosa. Rev Esp Cardiol. 2014;67:863-4.

9. Mogollón MV, Anguita MP, Aguado JM, Tornos P, Miró JM, Gálvez-Acebal J, et al. [Q fever endocarditis in Spain. Clinical characteristics and outcome.] Enferm Infecc Microbiol Clin. 2011;29:109-16.

10. García-Cabrera E, Fernández-Hidalgo N, Almirante B, Ivanova-Georgieva $R$ Noureddine M, Plata A, et al. Neurological complications of infective endocarditis: risk factors, outcome, and impact of cardiac surgery: a multicenter observational study. Circulation. 2013;127:2272-84.

11. Wilbring M, Irmscher L, Alexiou K, Matschke K, Tugtekin SM. The impact of preoperative neurological events in patients suffering from native infective valve endocarditis. Interact Cardiovasc Thorac Surg. 2014;18:740-7. 